\title{
Cornual Pregnancy Discovered on CT scan: A Case Report
}

\author{
Baadi $\mathrm{F}^{1^{*}}$, Gakosso $\mathrm{C}^{1}$, Rachid ${ }^{2}$, Oubahha ${ }^{2}$, Fakhir B ${ }^{2}$, Zouita $\mathrm{I}^{1}$, Jalal $\mathrm{H}^{1}$ \\ ${ }^{1}$ Radiology Department, Mother and Child Hospital, Mohammed VI CHU, Cadi Ayyad University, Marrakech \\ ${ }^{2}$ Obstetrics and Gynecology Department, Mother and Child Hospital, Mohammed VI CHU, Cadi Ayyad University, Marrakech
}

DOI: $10.36348 /$ sijog.2021.v04i01.004

| Received: 26.11.2020 | Accepted: 09.12.2020 | Published: 29.01.2021

*Corresponding author: Baadi $\mathrm{F}$

\section{Abstract}

Cornual pregnancy is uncommon among ectopic pregnancies. A diagnosis of cornual pregnancy remains challenging, and rupture of a cornual pregnancy causes catastrophic consequence due to massive bleeding. The purpose of this study is to determine the contribution of imaging in the early diagnosis and management of this rare entity, in order to avoid complications.

Keywords: Cornual pregnancy, US, CT, MRI.

Copyright $\odot 2021$ The Author(s): This is an open-access article distributed under the terms of the Creative Commons Attribution 4.0 International License (CC BY-NC 4.0) which permits unrestricted use, distribution, and reproduction in any medium for non-commercial use provided the original author and source are credited.

\section{INTRODUCTION}

Cornual pregnancy is a rare form of ectopic pregnancy, defined by the implantation of a gestational sac in the horn of the uterus, occurs in $2 \%$ of ectopic pregnancies [1].

The diagnosis of cornual pregnancy is most often showed by the pelvic ultrasound. Its discovery is late at the stage of rupture with the CT is very rare, it is more dangerous as it can lead to several complications. It may result in severe hemorrhage and shock, with mortality rates ranging between $2-2.5 \%$ [1].

Timor-Tritsh gives an ultrasound definition of pregnancy outside the corneal uterine according to 3 criteria: an empty uterine cavity with an eccentric sac to the right or left of the cavity; gestational content separated by more than $1 \mathrm{~cm}$ from the uterine cavity; and a peripheral myometrial crown $<5 \mathrm{~mm}$ thick [2].

In the meantime, the availability of MRI in the emergency room, transvaginal ultrasound coupled with the determination of plasma $\beta \mathrm{HCG}$ allows its diagnosis at an early stage [3].

\section{CASE REPORT}

A 24-year-old female patient with an episode of acute abdominal pain in the right hypochondrium, following 21 weeks amenorrhea to our accidental emergency.
This patient presents as obstetric history: fetal death in utero estimated at 34 weeks pregnant with preeclampsia.

According to her gynecologist performed an endo vaginal ultrasound in the emergency room, which confirmed a progressive intra uterine pregnancy.

The patient, initially taken care of by anaesthetist-intensive care, because she suddenly had an exacerbation of her pain in the right hypochondrium, accompanied by discomfort with high blood pressure and a urinary band of two proteinuria; evoke severe preeclampsia;

Thereafter the patient is complicated by a hypovolemic shock with a systolic blood pressure painfully maintained at $70 \mathrm{mmhg}$ despite an infusion of 2 liters of colloid solution.

Her clinical examination deteriorated and the patient became peritoneal on abdominal palpation. As a result, his treatment team has evoked a ruptured subcapsular hematoma of the liver; an abdominal ultrasound is performed in bed. Practiced under nonoptimal conditions, this eliminates the subcapsular hematoma of the liver and observes a hemoperitoneum of great abundance and a latero-deviated uterus on the right with empty vacuity line, extra uterine gestational sac on contact digestive loops with no detectable cardiac activity. 
Baadi F et al; Sch Int J Obstet Gynec, Jan. 2021; 4(1): 17-21

A the ruptured uterus of ectopic pregnancy has been evoked, but the medical team insists on supplementing with an abdominal CT given the patient's history of pre-eclampsia and pain in the right hypochondrium, the high blood pressure on admission and proteinuria.
An abdominal CT scan was performed in the emergency room, it objectified a rupture of the right anterior horn on a mono-fetal pregnancy with a large abundance of hemoperitoneum (Figures 1-6). Surgical exploration revealed a ruptured right corneal pregnancy with hemoperitoneum (Figures 7-9).

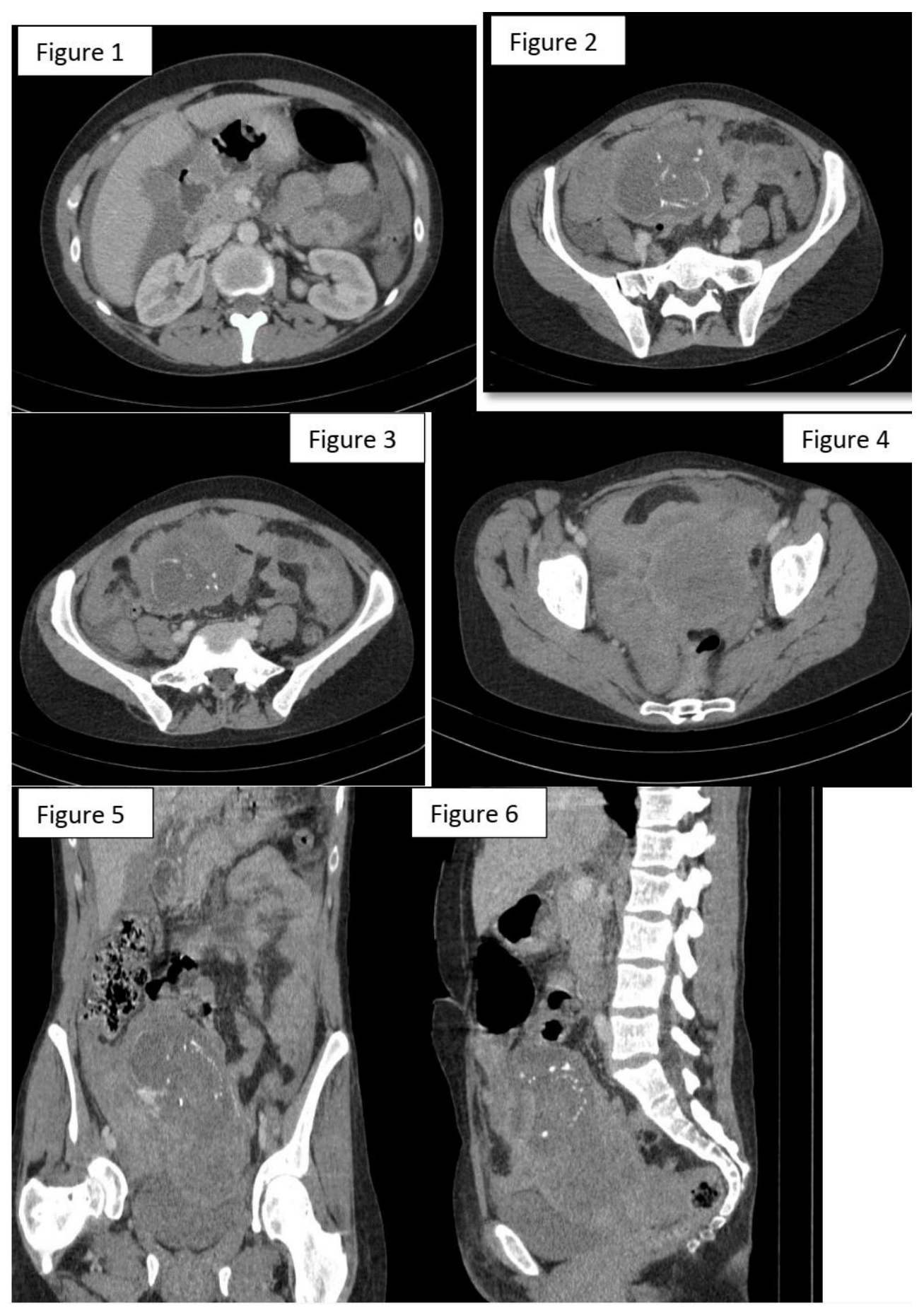

Figures 1-6: Abdominopelvic CT in axial, coronal and sagittal sections with injection of contrast medium: rupture of the right anterior horn on a mono-fetal pregnancy with a large abundance of hemoperitoneum 

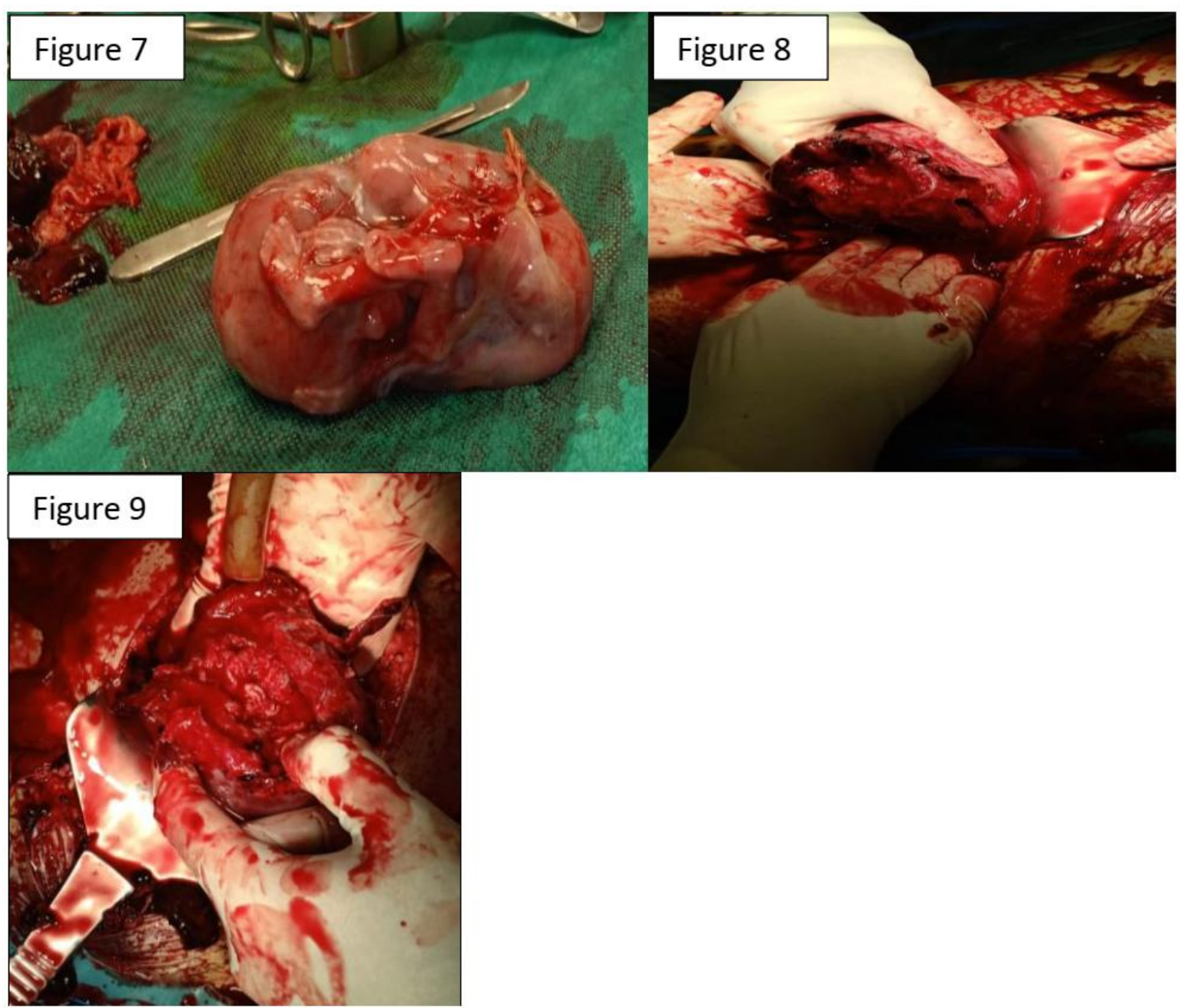

Figures 7-9: surgical exploration in the operating room finds a ruptured corneal pregnancy with large hemo-peritoneum, the patient benefited from an extraction of the pregnancy and a suture of the uterine horn then aspiration of blood and closure plan by plan.

\section{DISCUSSION}

A cornual pregnancy is defined as a pregnancy outside the uterus implanted in the rudimentary horn of a bicornuate uterus. By extension, this definition applies to pregnancies implanted in the horn of a bicornuate or septate uterus, some group together on this definition, the development of trophoblastic tissue on the stump remaining from a tube having been treated by salpingectomy [4].

According to Ardaens [5], cornual pregnancy should be defined as pregnancy outside the uterus of the uterine horn on uterine malformations (bicornuate uterus, unicornuate uterus, septate uterus). Conversely, the Anglo-Saxon authors $[6,7]$ extend this definition to all interstitial pregnancies.

The ultrasound diagnosis of cornual pregnancies is well known. According to Timor-Tritsch [7], there are three essential criteria: an empty uterine cavity, a gestational sac separated by more than $1 \mathrm{~cm}$ from the uterine cavity (interstitial line), a myometrial crown around this bag.
The risk factors for cornual ectopic pregnancies can be superimposed on the risk factors for tubal pregnancies. These include a history of ectopic pregnancy, united or bilateral salpingectomy, sexually transmitted infection, pregnancy through in vitro fertilization and maternal smoking [5].

In addition to these factors, there is a history of a bicornuate or pseudo-unicornuate uterus with a rudimentary tube, which are specific to corneal pregnancy.

In our case, none of these factors was known, even if we noted in the history a late abortion.

As with pregnancy outside the uterus, the diagnosis of cornual pregnancy is based on a combination of clinic, BHCG assay and pelvic ultrasound [6].

Cornual pregnancies maybe misdiagnosed as intrauterine, due to their partial implantation in the endometrium as in our case. 
The risk is the rupture, engaged the vital prognosis by massive externalized or intraperitoneal bleeding [3].

Ruptured cornual pregnancies are particularly haemorrhagic due to a rich corneal vascularization and greater myometrial distension through a more advanced pregnancy [7].

The risk of uterine rupture increases with the age of pregnancy, hence the urgent need for early diagnosis as in our case [6], thus rarely discovered late fortuitously during a routine obstetric ultrasound [8].

The appearance of cornual pregnancies is marked by the presence around the gestational sac of a myometrium which can allow its development until the 16th week of gestational age, exposing to sudden rupture and cataclysmic hemorrhage. Its prognosis is therefore more serious than conventional tubal ectopic pregnancy and requires an early and precise diagnosis before the rupture stage [9].

Computed tomography has no place in the positive diagnosis except in case of accidental discovery as in our case.

3D ultrasound also allows an accurate early diagnosis if interstitial pregnancy is suspected on 2D ultrasound [10].

MRI is currently the most effective imaging tool for diagnosing corneal pregnancies [9]. But it is not always available in emergency. For this, the diagnosis of cornual pregnancy is still based on a clinical summary, the result of the plasma HCG assay and transvaginal ultrasound.

Unlike tubal localization, there is no score to determine the success of medical treatment for cornual pregnancies. Thus, his treatment, whether medical or surgical, is not codified. He is guided both by the patient's hemodynamic condition and by the experience of the medical team [11].

The initial surgical treatment of interstitial pregnancy consisted of salpingectomy and corneal resection by laparotomy, this probably resulting from delayed diagnosis as in our case [12]. In addition to this radical treatment, several cases of conservative treatment have been reported [4].

In current practice, interstitial pregnancy is typically diagnosed at an early gestational age and before rupture, which leaves the opportunity to practice conservative medical or surgical treatment [13].

According to Soriano and his team, the best practice is to remove the interstitial pregnancy via cornuostomy with resection of the interstitial part of the tube if necessary and suturing of the hysterotomy [4]. With the advent of laparoscopy, trained teams are practicing conservative laparoscopic surgical treatment on hemodynamically stable patients [4].

Hysteroscopic removal of interstitial pregnancy has also been successfully described [14]. For any conservative treatment, the decrease in HCG should be monitored until complete negativation [4].

\section{CONCLUSION}

Cornual pregnancy, although rare, is an ectopic pregnancy of unusual location with a very serious prognosis that must be known at an early stage. Its diagnosis is often discovered late in the rupture stage and a life-threatening outcome for the patient. Endovaginal ultrasound by allowing its discovery at the early stage constitutes a very good means of diagnosis which in certain cases can be helped by the assay of plasma BHCG. If in doubt, magnetic resonance imaging, if available, will accurately state the diagnosis.

\section{RÉFÉRENCES}

1. Bouyer, J., Coste, J., Fernandez, H., Pouly, J. L., \& Job-Spira, N. (2002). Sites of ectopic pregnancy: a 10 year population-based study of 1800 cases. Human Reproduction, 17(12), 32243230 .

2. Timor-Tritsch, I. E., Monteagudo, A., Matera, C., \& Veit, C. R. (1992). Sonographic evolution of cornual pregnancies treated without surgery. Obstetrics and gynecology, 79(6), 10441049.

3. N'Goan-Domoua, A. M., N'Gbesso, R. D., \& Keita, A. K. (2011). Grossesse cornuale: une entité rare de grossesse extra-utérine. Imagerie de la femme, 21(2), 68-71.

4. Jourdain, O., Fontages, M., Schiano, A., Rauche, F., \& Gonnet, J. M. (2003). Prise en charge des autres ectopies annexielles (cornuale, interstitielle, angulaire et ovarienne). J Gynecol Obstet Biol Rprod. 32(Suppl. au n 7):3S93-3S100.

5. Capmas, P. (2015). Les grossesses ectopiques de localisation inhabituelle. Réalités en Gynecologieobstetrique octobre, 178.

6. Thouveny, A. (2013). Grossesses extra utérines de localisation rare à propos de 28 cas. Analyse des pratiques et proposition de protocoles thérapeutiques pour la Maternité Régionale Universitaire de Nancy.

7. Cheikhrouhou, H., Amoury, A., Sakhri, J., \& Ben, M. (2012). Traitement médical des grossesses interstitielles non rompues. La Tunisie Medicale, 90(5):421-423.

8. Guena, M. N., Nana, A. N., \& Amvene, S. N. O. (2017). Grossesse Cornuale Droite de Découverte Tardive et Fortuite à l'Échographie de Routine. Health Sciences and Disease, 18(4). 
9. Poncelet, É., Leconte, C., Fréart-Martinez, É., Laurent, N., Lernout, M., Bigot, J., ... \& Lucot, J. P. (2009). Aspect échographique et IRM de la grossesse extra-utérine. Imagerie de la Femme, 19(3), 171-178.

10. Júnior, E. A., Zanforlin Filho, S. M., Pires, C. R., Guimarães Filho, H. A., Massaguer, A. A., Nardozza, L. M. M., \& Moron, A. F. (2007). Three-dimensional transvaginal sonographic diagnosis of early and asymptomatic interstitial pregnancy. Archives of gynecology and obstetrics, 275(3), 207-210.

11. Fernandez, H., Lelaidier, C., Thouvenez, V., \& Frydman, R. (1991). The use of a pretherapeutic, predictive score to determine inclusion criteria for the non-surgical treatment of ectopic pregnancy. Human Reproduction, 6(7), 995-998.

12. Moawad, N. S., Mahajan, S. T., Moniz, M. H., Taylor, S. E., \& Hurd, W. W. (2010). Current diagnosis and treatment of interstitial pregnancy. American journal of obstetrics and gynecology, 202(1), 15-29.

13. Tulandi, T., \& Al-Jaroudi, D. (2004). Interstitial pregnancy: results generated from the Society of Reproductive Surgeons Registry. Obstetrics \& Gynecology, 103(1), 47-50.

14. Meyer, W. R., \& Mitchell, D. E. (1989). Hysteroscopic removal of an interstitial ectopic gestation. A case report. The Journal of reproductive medicine, 34(11), 928-929. 